\title{
Agile Testing and Critical Systems
}

\author{
Jørgen Bøegh ${ }^{1}$, Juan Garbajosa ${ }^{2}$, and Alex Rennoch ${ }^{3}$ \\ ${ }^{1}$ Beijing University of Posts and Telecommunications, No.10 Xitucheng Road, \\ Haidian District, Beijing 100876, China \\ ${ }^{2}$ Tehnical University of Madrid (UPM), Ctra. de Valencia, Km. 7. E-28031 Madrid, Spain \\ ${ }^{3}$ Fraunhofer FOKUS, MOTION, Kaiserin-Augusta-Allee 31, D-10589 Berlin, Germany \\ jorgen_boegh@yahoo.dk, jgs@eui.upm.es, \\ axel.rennochefocus. fraunhofer.de
}

\section{Workshop Description}

The need to count on critical systems is growing day by day. Together with traditional domains such as medical devices, automotive, railway, aeronautical, space and telecommunications, new applications and services are coming up every day. Terms such as ubiquitous, pervasive, or autonomic computing, products under the general umbrella of smart devices or the use of large wireless sensors networks indicate a clear trend in the increase of application complexity and dependency. The dependency of daily life on computers and computer based systems is growing up at a high rate, and testing is gaining importance at the same rate.

To perform a proper testing and validation of critical systems encounter a number of problems both from a technical and from a managerial point of view, also considering that the complexity of the underlying software is growing as well. Traditionally, critical systems are normally developed according to standards like IEC 61508, MIL 882 or ED 109, and emphasis is on well defined development processes. These systems usually have contractually specified requirements, and detailed documentation of all activities is very important. This approach is radically different from the usual agile development approach where focus is on customer collaboration, frequently changing requirements, and trust in individuals more than in documented quality activities. It is therefore a widely shared impression that the agile community and the critical system community live in completely different worlds.

However, there are mutual benefits of bringing the two communities together. For example, agile testing approaches can most likely bring many advantages to critical system testing. These possibilities are currently not well understood and exploited.

The high level topics of the workshop are the following:

- Shared values and principles in testing

- Quality Management goals

- Testing strategies

The workshop brings the agile and safety critical communities together, including representatives from both industry and academia. The workshop is organized in the context of the STV (System Testing and Validation) workshops, which were initiated in the year 2002. 